ras que facilitan la movilidad de las personas de mayor edad en esos países. (Reyes-Ortiz CA, et al. Cross-national comparison of disability in Latin American and Caribbean persons aged 75 and older. Arch Gerontol Geriatr. 2006;42:21-33).

\section{Revisión sistemática de estudios sobre la epilepsia en América Latina}

La epilepsia es la enfermedad neurológica grave más frecuente en el mundo, especialmente en los países en desarrollo, donde se detecta el $85 \%$ de los casos. Los datos de la incidencia de la epilepsia en América Latina muestran una gran heterogeneidad. En este trabajo se ofrecen los resultados de una revisión sistemática de los estudios retrospectivos y prospectivos basados en la comunidad que evaluaron la prevalencia y la incidencia de la epilepsia en adultos y niños de América Latina. Se analizaron los trabajos que emplearon cuestionarios validados previamente, encuestas y entrevistas y que se indizaron en las bases de datos bibliográficas MEDLINE, IMBIOMED y LILACS hasta julio de 2004.

Se encontraron 32 estudios de prevalencia y 3 de incidencia que cumplieron con los criterios de inclusión. La mediana de la prevalencia de toda la vida para todos los países fue de 17,8 por 1000 habitantes (mínimo: 6; máximo: 44,3), mientras que la mediana de la prevalencia de la epilepsia activa fue de 12,4 por 1000 habitantes (mínimo: 5,1; máximo: $57,0)$. No se encontraron diferencias significativas entre las prevalencias en hombres y mujeres.

Las tasas de incidencia por 100000 habitantes por año variaron entre 77,7 en Martinica y 190 en algunas partes de Ecuador. Esta gran heterogeneidad puede deberse a factores metodológicos, la calidad del diagnóstico, las particularidades de la población estudiada (edad, características socioeconómicas, lugar de residencia, etc.), los diferentes factores de riesgo e incluso a diferencias genéticas. Las variaciones en la prevalencia de esta enfermedad dentro de un mismo país son el reflejo de las diferencias entre las condiciones de vida de las diversas ciudades o regiones.

Los estudios epidemiológicos demostraron mayores tasas de prevalencia e incidencia de epilepsia en la población en general de América Latina que en los países del hemisferio norte. Es necesario identificar con mayor precisión las causas de esa heterogeneidad entre los países - y entre ciudades o regiones dentro de un mismo país-, mejorar los métodos de investigación y ampliar el conjunto de poblaciones estudiadas. Se deben escoger cuidadosamente las áreas de estudio y realizar estudios longitudinales que permitan tener una idea más clara no solamente de la incidencia, sino también de la morbilidad y mortalidad asociadas con la epilepsia. (Burneo JG, et al. Understanding the burden of epilepsy in Latin America: a systematic review of its prevalence and incidence. Epilepsy Res. 2005;66:63-74).

\section{Transmisión interpersonal del virus de los Andes}

El virus de los Andes (VA) es el único miembro americano del género Hantavirus que ha sido aislado de suero humano. Hasta el momento, seis cepas de VA han estado asociadas con casos de síndrome pulmonar por hantavirus en Argentina: la cepa VA sout en la región suroccidental; VA cent BsAs, VA cent Lec y VA cent Plata en la región central; y VA nort Orán y VA nort Bermejo en la región noroccidental. A pesar de que los roedores constituyen la vía principal de infección, se ha documentado la transmisión de la cepa VA sout de persona a persona.

En este trabajo se analizaron 13 casos de síndrome pulmonar por hantavirus que se detectaron en dos zonas con enfermedad endémica en Argentina durante 2002. En todos los casos se documentó la infección por hantavirus y en 10 de ellos se realizó la caracterización genética de la cepa responsable mediante el análisis nucleotídico de un fragmento de la región codificante G2 (desde la posición 2717 hasta la 2943).

Se identificaron cuatro conglomerados de casos según las relaciones epidemiológicas conocidas, entre los cuales se detectaron tres casos de transmisión de persona a persona. La comparación de las secuencias nucleotídicas de los virus de los cuatro conglomerados epidemiológicos demostró la identidad total entre los casos de un mismo conglomerado. La cepa VA cent BsAs se encontró en tres de los conglomerados, mientras que la cepa VA sout se encontró en tres casos de un conglomerado del suroeste del país, lo que constituye el primer informe de esta cepa en esa región argentina. Los casos de tres de los conglomerados tuvieron características clínicas similares entre sí, aunque diferentes de las de los casos encontrados en Buenos Aires. El período de incubación fue de 15 a 26 días.

Los resultados de este trabajo demuestran que la transmisión del virus de los Andes de una persona a otra es posible durante la fase prodrómica o inmediatamente después de ella. Las pruebas acumuladas hasta el momento indican que si bien los contactos esporádicos con personas en los primeros estadios del síndrome pulmonar por hantavirus no representan un riesgo de infección, se debe prestar atención a los contactos que ocurren en sitios cerrados, como los vehículos o el ambiente laboral. 